\title{
EL PROCESAMIENTO TRANSICIONAL DEL TERRORISMO DE ESTADO A VEINTE AÑOS DEL CASO PINOCHET
}

Juan Pablo Mañalich Raffo 


\section{JUAN PABLO MAÑALICH RAFFO}

Doctor en Derecho por la Rheinische FriedrichWilhelms-Universität Bonn y Licenciado en Ciencias Jurídicas y Sociales de la Universidad de Chile. Director y Profesor Titular del Departamento de Ciencias Penales de la Facultad de Derecho de la Universidad de Chile. 


\section{EL PROCESAMIENTO TRANSICIONAL DEL TERRORISMO DE ESTADO A VEINTE AÑOS DEL CASO PINOCHET}

\section{EL CASO PINOCHET BAJO EL DISEÑO TRANSICIONAL}

El 16 de octubre de este año marcó el transcurso de dos décadas desde que Augusto Pinochet fuera detenido en Londres en el marco del proceso de extradición encaminado a materializar el esfuerzo por su juzgamiento como responsable de crímenes de lesa humanidad. Al rememorar el que a mi juicio es el hito políticamente más significativo de lo que fuera dado en llamar la "transición chilena", me es imposible dejar de evocar la figura de quien fuera artífice del proceso de extradición activado en España y que tuvo al pueblo de Chile por aproximadamente un año y cinco meses pendiente de cómo se definía, en el Reino Unido, la situación procesal del otrora tirano y dictador. Joan Garcés, jurista, politólogo y asesor inmediato del Presidente Salvador Allende durante su gobierno constitucional, fue quien el día 5 de julio de 1996 presentó ante la Audiencia Nacional española la querella que condujo a que dos años, tres meses y once días más tarde, Pinochet fuera aprehendido con ocasión de su visita a Londres, cuando el entonces senador vitalicio se jactaba ante el mundo de la garantía de impunidad que el Estado transicional chileno le proporcionaba. Gracias a ello el pueblo de Chile tuvo ocasión de comprobar cómo podían quedar abruptamente modificados los términos que hasta aquel entonces todavía definían la "medida de lo posible".

Pero la razón por la cual la detención de Pinochet en Londres representa el hito más significativo de nuestro devenir transicional no se reduce a esta dimensión luminosa, de la cual Joan Garcés es, junto con tantos otros, protagonista heroico. Ese mismo hito nos proveyó de una inmejorable a la vez que dolorosa oportunidad para observar en vivo y en directo el despliegue de la estrategia política que delineó los estrechos contornos del proceso político chileno a contar del plebiscito del 5 de octubre de 1988. En el libro que recoge una larga entrevista concedida a la periodista Margarita Serrano, Edgardo Boeninger, el muy influyente ministro Secretario General de la Presidencia del gobierno de Patricio Aylwin, aportaba algunos puntos de vista que es prudente no soslayar a la hora de poner en perspectiva una dimensión menos luminosa del esfuerzo por someter a Pinochet al imperio de

1. Al respecto es elocuente el sucinto y sobrio prólogo incorporado, "cuatro décadas después", por Joan Garcés en su Allende y la experiencia chilena; véase Garcés (2013), pp.9 ss. 
la justicia. A propósito de lo que a su juicio había estado en juego en el plebiscito de 1988, Boeninger observaba lo siguiente:

"Estábamos convencidos de que el público no quería más guerra, quería paz. Tomamos una decisión en ese sentido, satisfacer a la gente. Y qué quería la gente: que le resolvieran los temas para que siguiera creciendo el país, así como los problemas sociales. Nosotros teníamos que hacer un máximo esfuerzo en lo social, seguir adelante con los beneficios económicos que Hernán Büchi ya había logrado durante cinco años; o sea, ya nos habían puesto la vara a cierta altura. Eso era lo decisivo. Y creo que en eso acertamos” (Serrano [2000], pp.184 s.).

Según Boeninger, entonces, lo que estuvo en juego para la Concertación de Partidos por la Democracia era asegurar, confiriéndole legitimidad democrática, la continuidad de lo que semana a semana el profesor Carlos Peña no sin admiración tematiza como el proceso de "modernización capitalista" que fuera impulsado por la dictadura. Unas pocas páginas más atrás, Boeninger destacaba que "lo de la Comisión Rettig fue una obra absolutamente de Aylwin”, para a renglón seguido recordar que en el entorno inmediato del primer presidente de la transición, y por influjo de su ministro de Justicia, Francisco Cumplido, se habría impuesto la convicción de que "los derechos humanos hay que sacarlos de la arena política" puesto que "entre el punto final de la derecha y las graves injusticias a las víctimas y, en consecuencia, la postura del Partido Socialista, no hay acomodo posible" (Serrano [2000], p.179).

Pero el "acomodo" fue encontrado y consistió en lo que podemos caracterizar como la estrategia de solución jurídica al "problema de los derechos humanos". A través de ella, el procesamiento político-institucional del terrorismo de Estado quedó, en lo fundamental, estrictamente relegado al foro judicial, de un modo que ha sido funcional a su reducción a un conjunto de crímenes singulares perpetrados por agentes individuales. Los ecos de semejante estrategia reduccionista llegaron a impactar, incluso, las iniciativas de carácter extrajudicial impulsadas por los gobiernos concertacionistas. Mientras el Informe Rettig validó la solución de compromiso consistente en reconocer violaciones de derechos humanos perpetradas por civiles en contra de funcionarios de las Fuerzas Armadas y de Orden, el documento que fijó el resultado alcanzado por la así llamada "Mesa de Diálogo", dado a conocer en junio del año 2000, prescindió del reconocimiento de una "política institucional y sistemática de violación de los derechos humanos", para validar, en cambio, una jerga que solo menciona "las graves violaciones a los derechos humanos en que incurrieron agentes de organizaciones del Estado durante el Gobierno Militar". 
Esta vocación por el eufemismo ha dado lugar a un escenario del todo favorable para quienes apoyaron sostenida y entusiastamente al régimen que desplegó esa violencia criminal, pero sin llegar a "mancharse las manos de sangre", y que hoy esgrimen esta precisa circunstancia para separarse, cobardemente, de esa misma violencia.

Sobre este trasfondo, no es difícil racionalizar el comportamiento que el gobierno del Presidente Frei Ruiz-Tagle mostró a través de su orquestado esfuerzo por lograr la repatriación de Pinochet desde Londres y así impedir que se materializara la extradición del tirano y dictador requerida por el Estado español. La transición chilena fue pactada sobre el entendido de que Augusto Pinochet no enfrentaría a la justicia por los crímenes perpetrados por su régimen y lo ocurrido a partir del 16 de octubre de 1998 logró poner radicalmente en entredicho ese auténtico axioma transicional. Esto quizás logre explicar la tenacidad con la que, a través de la resuelta operación de su entonces ministro de Relaciones Exteriores, el socialista José Miguel Insulza, ese mismo gobierno actuó para inhibir el sometimiento de Pinochet a la jurisdicción de un tribunal foráneo. Ello resulta difícil de controvertir si recordamos que, una vez fracasado el intento de impedir que la Cámara de los Lores reconociera la legitimidad preferente de la jurisdicción chilena, la estrategia gubernamental se travistió en una invocación de razones humanitarias en atención a la situación de salud que habría afectado al imputado, para lograr que el ministro del Interior británico, Jack Straw, dispusiera la denegación de la extradición, posibilitando así la repatriación de Pinochet.

\section{EL IMPACTO EN EL PROCESAMIENTO JUDICIAL DEL TERRORISMO DE ESTADO}

Este desenlace ciertamente no privó de impacto al sometimiento de Pinochet al proceso de extradición en el desenvolvimiento posterior de la ya referida estrategia de solución jurídica al "problema de los derechos humanos". Pues es difícil explicar, en términos de sociología judicial, el giro experimentado hacia fines del primer lustro de la década del 2000 por la praxis de los tribunales superiores de justicia chilenos, y en particular de la Corte Suprema, en lo tocante a la incorporación de premisas extraídas del derecho internacional a los esquemas de justificación de las decisiones de condena pronunciadas en este ámbito si prescindimos de la inequívoca demostración de su pertinencia que trajo consigo el icónico "caso Pinochet"2.

2. Al respecto, Mañalich (2010), pp.155 ss., 174 ss., con una consideración crítica de la irrelevancia que el discurso del derecho internacional de los derechos humanos ha tendido a atribuir al carácter de auto-amnistía que exhibe la amnistía establecida por el DL 2191. 
Ese giro estuvo en lo fundamental determinado, en primer lugar, por el reconocimiento de la ineficacia del Decreto Ley 2191, de amnistía, de 18 de abril de 1978, como base de una extinción de la responsabilidad referida a hechos punibles que el derecho internacional reviste del estatus de crímenes contra la humanidad. Ello hizo posible que la judicatura chilena dejara atrás las dos vías de elusión del obstáculo representado por esa auto-amnistía de las que sucesivamente se había valido, hasta entonces, para llevar adelante los procesos referidos a hechos perpetrados con anterioridad al 10 de marzo de $1978^{3}$. La primera de esas dos vías de elusión consistió en la validación de la así llamada "doctrina Aylwin", conducente a que bajo una determinada interpretación de las disposiciones pertinentes del Código de Procedimiento Penal, la extinción de responsabilidad fundada en esa amnistía y resultante en el pronunciamiento del respectivo sobreseimiento definitivo, recién hubiera de ser declarada al cabo del sumario correspondiente y solo si hubiese quedado determinada la identidad de los responsables ${ }^{4}$. Por su parte, la segunda vía de elusión consistió en el recurso a la así llamada "tesis del secuestro permanente", expresión pleonástica a través de la cual se designaba un argumento dirigido a invertir la carga de la prueba en referencia a la comprobación del momento de cesación de la privación de libertad sufrida por la víctima de un secuestro constitutivo, en el sentido del derecho internacional, de una desaparición forzada de persona ${ }^{5}$.

El reconocimiento jurisdiccional de la invalidez del DL 2191 bajo el orden jurídico internacional no impidió que el Estado de Chile fuera condenado por la Corte Interamericana de Derechos Humanos a través de su sentencia recaída en el caso "Almonacid Arellano" de 26 de septiembre de 2006, por haber dado aplicación judicial al decreto ley en cuestión, así como por no haber dado lugar a su anulación o derogación por vía legislativa. Pero ese reconocimiento sobreviniente de la invalidez del decreto ley en cuestión sí modificó drásticamente los términos del debate forense.

El segundo aspecto del giro jurisprudencial aquí considerado estuvo constituido por el desconocimiento de la operatividad de la prescripción de la acción penal como causa de extinción de la responsabilidad ${ }^{6}$. Como es sabido, este paso ha quedado simultáneamente marcado por la sorprendente tendencia de la segunda sala de la Corte Suprema a validar, ocasionalmente, la tesis de la procedencia de la híper-atenuante prevista en el art. 103 del Código Penal, coloquialmente etiquetada

3. Para lo que sigue, véase Mañalich (2010), pp.157 ss., 162 ss.

4. Sobre ello, véase Couso y Mera (2011), pp.121 ss.

5. Véase Couso y Mera (2011), pp.126 ss.

6. $\mathrm{Al}$ respecto, véase Mañalich (2010), pp.205 ss., 233 s. 
como "media prescripción", ${ }^{7}$ en términos que la desacreditan como tribunal de derecho. Pues hasta el día de hoy los ministros que se inclinan a favor de tal posición -conocida como "doctrina Dolmetsch" en razón del ministro a quien se atribuye su elaboración ${ }^{8}$ - no han logrado explicar, porque de hecho no es posible explicar, que un plazo de prescripción que se declara no correr en razón del carácter imprescriptible de la respectiva acción penal, pueda, no obstante, ver transcurrir más de la mitad de su duración?.

Esta aberración jurídica ha llegado a ser defendida mediante el recurso a una preocupante diferenciación de lo que exigiría el derecho aplicable, por un lado, y lo que demandaría la impartición de auténtica justicia, por otro, la cual no sería concebible "si no hay misericordia" 10 . Si no fuera por la inmunidad con la que el art. 324 del Código Orgánico de Tribunales protege a los integrantes de la Corte Suprema frente a tal título de punibilidad, el asunto admitiría ser examinado bajo la pregunta de si, por esa vía, pudiera configurarse una prevaricación en la forma de un fallar a sabiendas "contra ley expresa y vigente en causa criminal", según reza el art. 223, Nº1, del Código Penal.

Pero nótese que del hecho de que semejante especulación jurídica tenga perfecto sentido no se sigue, de manera alguna, que haya tenido sustento específico la acusación constitucional recientemente dirigida contra tres ministros de esa misma sala, en referencia a la manera en que, pronunciándose sobre la apelación de resoluciones que habían denegado las respectivas acciones de amparo interpuestas a favor de condenados por delitos constitutivos de crímenes de lesa humanidad, ellos entendieron satisfechas las condiciones de las cuales depende, bajo el Decreto

7. Para una consideración de los primeros pronunciamientos emitidos por la Corte Suprema en esa dirección, véase Fernández y Sferrazza (2009), pp.184 ss.

8. Sobre ello, véase por ejemplo Matus (2012), quien caracterizaba la doctrina en cuestión "como una suerte de 'compromiso' entre los afanes de 'justicia absoluta' y 'perdón absoluto"'.

9. En otros pronunciamientos de la Corte Suprema, la sola consideración de que la aplicabilidad de la regla del art. 103 se encuentra condicionada por el requisito de que haya "ya transcurrido la mitad del [tiempo] que se exige" para la correspondiente prescripción es tenida por suficiente para concluir que tal regla resulta inaplicable tratándose de hechos punibles, a cuyo respecto la correspondiente acción penal se tiene por imprescriptible, lo cual lleva a una desestimación del reconocimiento de la híper-atenuante a favor del condenado respectivo. Véase, por ejemplo, la sentencia de 24 mayo de 2016, recaída en causa rol No 37035-15, redactada por el ministro Jorge Dahm, en su considerando $11^{\circ}$. Que en este punto el problema sigue dividiendo a quienes integran la segunda sala queda de manifiesto en atención al tenor del voto de prevención emitido por el ministro Carlos Künsemüller y el abogado integrante Jean Pierre Matus.

10. Así se expresaba el propio ministro Dolmetsch en su entrevista concedida a la revista Caras, publicada con fecha 5 de mayo de 2016, disponible en: http://www.caras.cl/politica/hugodolmestch-hay-justicia-sin-misericordia (última consulta: 22 de octubre de 2018). 
Ley 321, el otorgamiento de una libertad condicional. A través de la acusación se pretendía reprochar a esos jueces que, respecto de un asunto que el pacto de la transición deliberadamente redujo a un tópico de controversia judicial, ellos se hayan comportado como de hecho se comportan cada vez que un caso particular llega a su conocimiento, a saber: demostrando su renuencia a contribuir a uniformar los criterios que deberían gobernar la aplicación judicial del derecho. Como acertadamente han observado Couso y Mera:

"La primera explicación cultural de esta situación es la vocación de la CS por realizar la 'justicia material' en el caso concreto, lo que implica la revisión de la decisión de fondo, más allá de la enmienda de algún error de derecho específico cometido por la sentencia recurrida, y la disposición a abandonar, en caso necesario, la doctrina que ha sostenido en fallos anteriores no a través de una tesis nueva -que en adelante se aplicará consistentemente- sino con una interpretación ad hoc de la ley por esa sola vez. Esta inclinación retrospectiva se explica en parte por una 'deformación profesional' de sus ministros, formados como jueces de instancia y por lo tanto preocupados precisamente de hacer justicia en el caso concreto. Su renuencia a citar sus propios precedentes también da cuenta de la falta de orientación prospectiva de la Sala Penal. No es de extrañar que las cortes de apelaciones sólo en contadas ocasiones citen los precedentes de la Sala Penal, sea que los sigan o se aparten de ellos" (Couso y Mera [2011], p.37).

La existencia de vaivenes “jurisprudenciales”, según cómo quede integrada su segunda sala, es algo a lo que la Corte Suprema nos tiene suficientemente acostumbrados. Y que en su conocimiento de los casos que culminaron en el pronunciamiento de los fallos que motivaron la acusación constitucional, la segunda sala en efecto se haya comportado como un mero tribunal de instancia, es algo que no puede merecer reparo alguno si se advierte que, tratándose del ejercicio de una acción de amparo, la competencia de la Corte Suprema es, precisamente, la de un tribunal de apelación ${ }^{11}$. La ingente variabilidad de los pronunciamientos que ella emite es un rasgo ciertamente preocupante, pero de ninguna manera excepcional, del comportamiento jurisdiccional de la Corte Suprema. Se trata, antes bien, de un aspecto definitorio de una praxis judicial que en general se distingue por mostrar un déficit de autoconsciencia en la concatenación de pronunciamientos sucesivos acerca de los mismos problemas.

11. Ello, según lo establecido en el No 4 del art. 98 del Código Orgánico de Tribunales en relación con el art. 21 de la Constitución Política de 1980. 


\section{LA TRIVIALIZACIÓN JURÍDICA DEL TERROR}

El confinamiento del procesamiento político-institucional del terror dictatorial a la rutina de una aplicación técnicamente desprolija de la legislación penal ordinaria es, en buena medida, una marca de éxito de la estrategia transicional honestamente reseñada por Boeninger. Y tal como intentara mostrarlo en mi libro Terror, pena y amnistía, el resultado más sobresaliente de esa estrategia consiste en una trivialización del terror ${ }^{12}$. Quisiera sustentar este último aserto a través de una breve reflexión acerca de la funcionalidad que es posible atribuir a la materialización de una reacción punitiva como consecuencia jurídica de un hecho punible.

En nuestro contexto cultural, el debate entre las posiciones que compiten por ofrecer una justificación teóricamente adecuada de la pena estatal tiende a estar dominado por concepciones que identifican la finalidad legitimadora de la punición con algún objetivo de carácter preventivo. En este sentido, lo que distingue a cualquier teoría de la prevención es el esfuerzo por caracterizar el mecanismo de la punición como una herramienta de evitación de crímenes futuros. Este punto de vista también impacta, como es obvio, el debate más específico acerca de la justificación de la pena estatal al interior de procesos de justicia transicional; aquí se trataría ante todo de inhibir, a través de una estrategia de prevención general, la perpetración futura de crímenes por parte de, o con la aquiescencia de, regímenes autoritarios o totalitarios. El problema fundamental que aqueja a estas concepciones radica en su subordinación de la justificación de la pena estatal a la producción de efectos de índole sociológica o psicológica, cuya conexión con la imposición y ejecución de la pena respectiva es puramente contingente, en cuanto dependiente de la satisfacción de condiciones empíricas que la punición como tal jamás puede garantizar.

Más realista, me parece, es asumir desde el comienzo una concepción de la pena estatal bajo la cual esta se presenta como un artefacto ideológico cuya funcionalidad propiamente jurídica consiste en servir como un mecanismo de reafirmación -simbólica o comunicativa- de la autoridad del derecho estatalmente producido y aplicado ${ }^{13}$. Desde esta perspectiva, la reafirmación de la autoridad del derecho quebrantado no es algo que hubiera de tener lugar, contingentemente, a consecuencia de la punición, sino que ella se encuentra constitutivamente determinada por la satisfacción de las condiciones institucionales de las cuales depende que la respectiva reacción al quebrantamiento imputable del derecho exhiba el estatus de una pena merecida. O formulando el punto en los términos de la teoría de

12. Mañalich (2010), pp.9 ss., 29 ss.

13. Sobre esto, véase Mañalich (2018), pp.38 ss., 50 ss. 
los actos de habla: la reafirmación de la autoridad del derecho quebrantado no se corresponde con un eventual efecto perlocutivo de la punición en cuanto instancia de acción simbólicamente mediada, sino que es la marca de su distintiva fuerza ilocutiva, la cual se encuentra implícitamente codificada en la respectiva instancia de irrogación de un mal que es, a su vez, ideológicamente interpretada como una reacción punitiva merecida.

El punto de vista así (apenas) delineado tiene importancia para dar cuenta de una particularidad del debate acerca de la justificación del castigo en contextos transicionales, que consiste en el énfasis puesto en el desiderátum de la superación de la impunidad. Un rasgo distintivo de los procesos de justicia transicional está constituido por la pretensión de revertir la inmunidad frente al castigo que las y los responsables del despliegue de violencia criminal como método de dominación política han logrado conferirse a sí mismos. En tal medida, la punición de los responsables de tales crímenes logra refutar, retrospectivamente, su pretensión de estar por encima de la fuerza vinculante del derecho.

Lo que no podemos perder de vista, con todo, es que la descripción recién ofrecida reclama ser verdadera de toda instancia de punición jurídica de un modo que resulta ser irremediablemente insensible a la distinción entre lo que podríamos llamar "criminalidad regular" y "criminalidad excepcional". Esto se sigue de que la operación del derecho solo está en condiciones de procesar aquello que la propia operación del derecho vuelve jurídicamente conmensurable. Pues como ha sugerido Christodoulidis, "el derecho pre-estructura su pasado de un modo tal que el pasado es moldeado en términos conmensurados con su superación, i.e., con su propia proyección de expectativas hacia el futuro" (Christodoulidis [2001], p.222).

Por esta vía, Christodoulidis ofrece un muy perspicaz señalamiento de los límites a los que puede verse sometido el esfuerzo por la superación de un pasado políticamente insoportable cuando ese esfuerzo pretende quedar estrictamente confinado a cauces jurídicos. Semejante superación tendría que consistir en la demostración de la inconmensurabilidad entre el pasado y el presente de la respectiva comunidad política. Pero es exactamente esto, la producción de un quiebre en la auto-comprensión de la respectiva comunidad política, lo que no puede tener lugar a través de la operación del derecho. Pues cada aplicación del derecho encierra el alegato implícito de una congruencia entre ayer y hoy, entre el tiempo (pasado) del hecho juzgado y el tiempo (presente) del juzgamiento del hecho.

¿Qué significaría hacer de la experiencia histórica del terrorismo de Estado un fenómeno jurídicamente conmensurable? La respuesta a esta pregunta es exactamente aquella que, en el contexto de la transición chilena, se ha dado a la pregunta por la significación de la actividad desplegada por las agencias represoras de la dictadura cívico-militar, a saber: una actividad consistente en la perpetración 
masiva de crímenes de particular gravedad. Semejante descripción conlleva una trivialización. Porque la transformación de (a lo menos) una parte del aparato estatal en un aparato criminal supone ya una supresión de las condiciones de la juridicidad de la praxis de ese Estado, sin que esa pérdida de juridicidad pueda ser radicalmente tematizada, sin embargo, bajo el derecho de ese Estado.

Políticamente, que en el marco del Chile transicional nuestro presente esté reconciliado con nuestro pasado significa que nuestro actual estado de cosas es (re) presentado como el resultado del ejercicio del terror dictatorial como vehículo criminal para la instauración fundacional del orden que hasta hoy habitamos ${ }^{14}$. Aquí es pertinente dar nuevamente la palabra a Joan Garcés:

“[...] el 11 de septiembre de 1973 no fue un cuartelazo latinoamericano más. Era una etapa en la deliberada, sistemática, científica destrucción de un Estado democrático, de las libertades y soberanía de su pueblo. La solemnización del sometimiento de la sociedad fue plasmada en la Constitución militar de 1980. El país latinoamericano continúa sometido, aun después de ser apartado el Dictador del Gobierno y reemplazado en 1989 por una Democracia Cristiana en manos del sector que en 1973 dirigió la insurrección y la destrucción del Estado republicano -el agrupado en torno de Patricio Aylwin y Frei, que veinte años después disponía de un instrumento auxiliar 'legalizado' que no tuvo en 1970: el control conferido a los jefes militares sobre las instituciones representativas del Estado, Parlamento y Ejecutivo incluidos, por la 'Constitución' impuesta desde 1980”' (Garcés [2012], pp.146 s.).

Para concluir, cabría apuntar que el devenir de la (re)presentación constitucionalmente sublimada de la instauración criminal de nuestro actual orden institucional ha traído consigo, ulteriormente, una privatización de la disputa acerca del significado del terrorismo de Estado, como si se tratara de una disputa en la que se enfrentan los victimarios y sus apologistas, por un lado, y las víctimas y sus deudos, por el otro. Esto es algo que comprobamos cada vez que las cámaras y los micrófonos se redirigen, de cuando en cuando, hacia quienes han luchado por impedir que las víctimas empíricas del terror vuelvan a desparecer, esta vez en el olvido, para que ellos manifiesten una disposición favorable a perdonar a los perpetradores, como si aquí estuviéramos ante delitos de acción penal privada ${ }^{15}$.

14. Para una presentación más exhaustiva de este punto de vista, véase Mañalich (2016), pp.179 ss.

15. Tratándose de los cuales, y según lo establece el art. 93, № 5, del Código Penal, el "perdón del ofendido" opera como una causa de extinción de la responsabilidad. 
Cuando esto ocurre, somos testigos de un lapsus que deja entrever que el pretendido consenso público que las respectivas condenas tendrían que simbolizar es, más bien, una impostura que esconde el hecho de que la transición los dejó solos, administrando un padecimiento que, en retrospectiva, se nos presenta como el costo de la celebrada modernización capitalista que esa misma transición legitimó. 


\section{REFERENCIAS}

Christodoulidis, Emilios (2001): “Law's Immemorial”, en Christodoulidis, Emilios y Veitch, Scott (eds.), Lethe's Law. Justice, Law and Ethics in Reconciliation. Oxford y Portland, Hart.

Couso, Jaime y Mera, Jorge (2011): Precedentes y justicia penal. Santiago, Ediciones Universidad Diego Portales.

Fernández, Karina y Sferrazza, Pietro (2009): "La aplicación de la prescripción gradual del delito en causas sobre violaciones de derechos humanos". Anuario de Derechos Humanos 5, pp.183-192.

Garcés, Joan (2012): Soberanos e intervenidos. Estrategias globales, americanos y españoles, $4^{\mathrm{a}}$ ed. Madrid, Siglo XXI.

Garcés, Joan (2013): Allende y la experiencia chilena. Las armas de la politica, $2^{\mathrm{a}}$ ed. Madrid y Santiago, Siglo XXI y Hueders.

Mañalich, Juan Pablo (2010): Terror, pena y amnistía. Santiago, Flandes Indiano.

Mañalich, Juan Pablo (2016): “Terror, memoria y archivos”. Anuario de Derechos Humanos 12, pp.173-189.

Mañalich, Juan Pablo (2018): Estudios sobre la fundamentación y la determinación de la pena. Santiago, Thomson Reuters.

Matus, Jean Pierre (2012): "El fin de la doctrina Dolmetsch". El Mercurio Legal, 30 de julio de 2012.

Serrano, Margarita (2000): La igual libertad de Edgardo Boeninger. Santiago, Uqbar. 